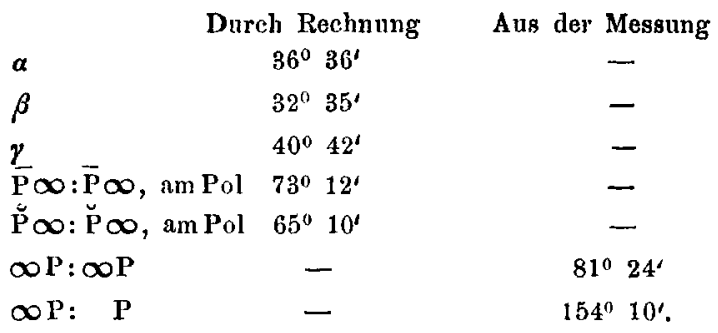

Den chemischen Theil dieser Untersuchungen habe ich im hiesigen academischen chemischen Laboratorium ausgeführt. Für die mir hier so oft erwiesenen collegialischen und freundschaftlichen Unterstützungen von den Herren Professor Will und Dr. Th. Engelbach spreche ich meinen Dank aus.

\title{
Chlornatrium in Hexakisoctaëdern; krystallographische Notiz von Demselben.
}

Es ist eine schon von Hauy (vgl. Traité de Minéralogie II, 193) mitgetheilte Erfahrung, dafs Kochsalz aus einer Lösung in Urin krystallisirt die Octaëderform annimmt. Um gröfsere Octaëder des Kochsalzes zu ziehen habe ich etwa 1 Pfund dieser Substanz in Urin aufgelöst und einer sehr langsamen Verdunstung überlassen. Nach etwa einem halben Jahre hatten sich am Boden des Gefälses bis einen halben Zoll Axenlänge haltende octaëderähnliche Formen ausgeschieden, welche bei genauerer Besichtigung als deutlich ausgebildete Hexakisoctaëder erkannt wurden. Diese rein ausgebildeten Krystalle waren klar und durchsichtig, mit glänzenden, wiewohl nicht scharf spiegelnden Flächen. 
Aus annähernden Messungen der Kanten dieser Form ergab sich für sie das Parameterverhältnifs $=a: 6 / 5$ a: $5 / 3$ a $=6 / 505 / 3$ (vgl. Fig. 10 auf Tafel I).

Es wurde gefunden :

$\begin{array}{lcc} & \text { Durch Messung } & \text { Durch Rechnung } \\ \text { A, die längste Kante } & 166^{\circ} & 166^{\circ} 46^{\prime} \\ \text { B, die mittlere Kante } & 130^{\circ} 20^{\prime} & 130^{\circ} 30^{\prime} \\ \text { C, die kürzeste Kante } & 171^{\circ} & 170^{\circ} 34^{\prime} .\end{array}$

Bei mehrfacher Wiederholung desselben Versuchs habe ich dieselben Gestalten auch in kürzerer Zeit erhalten. Scheinbar reine Octaëder zeigten sich auf ihren Flächen stets aus einer Summe mehr oder weniger mit ihren hexaëdrischen Ecken hervorragender Hexakisoctaëder zusammengesetzt.

Diese Hexakisoctaëder von neuem in reinem Wasser aufgelöst schieden sich in Combination mit untergeordnetem Würfel wieder aus.

\section{Untersuchungen über die Amylalkohole; von M. Berthelot*).}

Es giebt zwei allgemeine Methoden, aus Kohlenwasserstoffen Alkohole entstehen zu lassen : Die eine besteht darin, Sauerstoff auf den Kohlenwasserstoffen $\mathrm{C}_{2 \mathrm{n}} \mathrm{H}_{2 \mathrm{n}+2}$ zu fixiren; die andere darin, die Elemente des Wassers auf den Kohlenwasserstoffen $\mathrm{C}_{2_{\mathrm{L}}} \mathrm{H}_{2 \mathrm{n}}$ zu fixiren. Nach dem ersteren Verfahren habe ich das Sumpfgas $\mathrm{C}_{2} \mathrm{H}_{4}$ zu Methylalkohol $\mathrm{C}_{2} \mathrm{H}_{4} \mathrm{O}_{4}$ umgewandelt, unter vorgängiger Umwandlung des ersteren

*) Compt, rend. LVI, 700 . 